\title{
Heat Transfer Rates in Wall Bounded Shear Flows near the Jamming Point accompanied by Fluid-Particle Heat Exchange.
}

\author{
Thomas Forgber • Stefan Radl
}

Received: 26.8 .2016

\begin{abstract}
We investigate the heat transfer rates through a contact network of a sheared granular material using the Discrete Element Method (DEM). The tool ParScale enables us to study intraparticle transport processes, and the DEM solver LIGGGHTS is used to carry out the simulations. Our setup enables us to investigate a plethora of dimensionless parameters in order to picture different phenomena occurring near the jamming point (e.g., solidification) of a sheared granular material. Thereby, our main outcome is the quantification of thermal fluxes which occur due to (i) conduction in case of particle-particle collisions, as well as (ii) convection due to random particle motion. Also, the transferred heat between the particles and the ambient fluid is analyzed. In addition, we analyse the phenomena of crystallisation, and its effect on the thermal transport rate in the granular material. Again we exploit the measured thermal fluxes to decide whether a system is crystallized or not. Furthermore, we demonstrate that the ordering process, and hence the heat transport rate, can be affected by adjusting the coefficient of friction of the granular material.
\end{abstract}

\section{Introduction}

Sheared particle beds occur in a large variety of industrial and geological applications. Many researchers have investigated related phenomena like critical jamming behaviour, rheological models, crystallization and stress relaxation over the past years. Early work was focussed on dilute flows with fluid-like behaviour (inertial flows) where kinetic theories can be applied (Garzo et al. [1], Lun et al. [2], Jenkins et al. [3], Johnson et al. [4]). On the contrary it has been found that dense flows (quasi-static) can be described using plasticity models. More recent publications aim on rheology models around the transition regime in flows that involve frictionless spheres (Hatano [5], Nordstrom et al. [6], Seth et al. [7], Otsuki et al. [8]). Scaling of the normal and shear stress tensor has been used with success for a wide range of volume fractions and dimensionless shear rates to collapse the rheological data.

Drawing back to industrial applications, granular matter is typically described as an ensemble of frictional, stiff spheres, and past work was mostly focussed on the critical jamming and rheology behaviour of these materials (Otsuki et al. [9], Zhang et al. [10], Song et al. [11], Majmudar et al.

T. Forgber, S. Radl

Institute of Process- and Particle Engineering, Inffeldgasse 13/III,8010 Graz

Tel.: +43/316/873 30412

E-mail: thomas.forgber@tugraz.at 
[12], Tighe et al. [13], Vescovi et al. [14]). Referring to the original jamming diagram by Liu et al. [15], the influence of the friction coefficient was originally not taken into account. However, more advanced theories consider frictional, jammed granular materials, and were developed based on the interest to more application-oriented problems (Luding [16]). This work also triggered the development of advanced jamming diagrams (Ciamarra et al. [17], Brown et al. [18]). Clearly, most models from literature rely on the contact pressure or related quantities to determine the state of the system (e.g., unjammed, jammed or in the ordering process). In fact, the contact pressure is very sensitive to the applied modelling technique and system, and hence is certainly suitable from a rheological point of view to characterize jamming.

The above mentioned work relied on the Molecular Dynamics (MD) or Discrete Element Method (DEM), and mostly focussed on the rheological behaviour of granular matter in a fully periodic domain. Typically, a rather rudimentary approach for the local particle temperature distribution was adopted, or the system was treated as isothermal. With the focus on predicting local temperatures in chemical reactors for, e.g., $\mathrm{CO}_{2}$ absorption, or biomass combustion, a reliable model is still missing in literature. Only a few researchers made the attempt to model thermal fluxes in sheared beds: for example, Mohan et al. [19] showed the existence of different regimes of conductive and convective liquid transport in a sheared particle bed, and similarities between the transport of thermal energy and liquid adhering to the surface of the particles have been discussed. Thereby, a dimensionless shear rate and a Peclet number were identified as the key dimensionless parameters. Unfortunately, the work of Mohan et al. [19] relied on the assumption of zero heat flux to the ambient fluid, critically limiting the applicability of their conclusions. Rognon et al. [20], [21] did not even attempt to model the transferred heat from the particle to the ambient fluid (e.g., air), but was successfuly in formulating scaling laws for conductive heat fluxes in the system. As previous work was mostly performed using the Lees-Edwards boundary conditions [22], it is also unknown how a application-oriented boundary (e.g., flat walls) influences the thermal fluxes. This is, however, of great significance since the heat exchange with walls often dominates the temperature distribution within a reactor, and hence its performance. Even the effect of walls on the scaling of, e.g., the convective flux due to the random movement of the particles [21], is still unknown.Also, there is currently no hope to reliably predict thermal fluxes to, or near walls in the context of continuum models, even in case we assume isothermal particles.

In summary, there is still very little quantitative knowledge about the thermal behaviour of a wall-bounded particle bed. This is especially true for granular material that consists of particles with a non-uniform temperature profile, and which exchanges heat with the ambient fluid. Clearly, a thorough understanding of the relative rates of (i) the transferred heat to the ambient fluid, (ii) the convective heat transport in the granular material, as well as (iii) the heat that is transported via conduction is still missing in literature. Also, a critical assessment of the influence of friction coefficient and diameter distribution on the effect of crystallisation and jamming is needed. Most importantly, combining recent results on the jamming behavior, as well as on crystallization effects with recently developed methods to study thermal transport in these system seems necessary.

It is our goal improve this situation by coupling DEM-based simulations to a tool that is capable of resolving intra-particle temperature profiles. Our tool ParScale (Radl et al. [23], Forgber et al. [24], Forgber et al. [25]) allows us to efficiently solve a one-dimensional heat conduction equation within each particle. By means of such coupled DEM-based simulations a generally aplicable continuum model for predicting thermal fluxes in granular materials can be established. Such a model is of utmost importance for the correct selection of an appropriate simulation model for a specific fluid-particle heat transport problem, e.g., toe model a nonisothermal heterogeneous reactor. In our present contribution we analyse temperature gradients by probing an adequate space of dimensionless parameters, most important the Biot and Peclet 
number, in a wall-bounded shear flow. This allows us to draw conclusions that are applicable to a wide range of real-world applications, and help to lay the foundation for an advanced continuum model of non-isothermal granular flows.

Our manuscript is structured as follows: we first (in Section 2) explain our simulation method and present an overview of all equations solved, including the known and unknown simulation parameters. In the following Section 3 we identify and define the most influential dimensionless parameters which enables us to scale our results, and apply the outcome of our study to a wide range of relevant industrial applications. In Section 4 we present simulation results including schematic drawings of our wall bounded shear box set-up with studied parameter ranges. Section 5 summarizes our study and outlines ideas for follow-up work. Note that we included a Nomenclature (Section 6) at the end of our manuscript where all symbols are defined. 


\section{Simulation method}

As in earlier work (Forgber et al. [25]), we do not solve any equation for the fluid phase (e.g. ambient fluid) and assume a fixed heat transfer coefficient $\alpha$, as well as a fixed ambient fluid temperature $t_{f}$. In the final application of our models, these quantities are provided by an external Finite Volume Method (FVM) solver, and hence are known. We summarized all equations solved in Tab. (1), as well as all unknown particle parameters in Tab. 2.

\subsection{Particle flow model}

In order to correctly account for particle movement and particle-particle interactions we rely on the open-source software package LIGGGHTS [26], [19] which is a state of the art tool to perform Discrete Element Method-based simulations. We use this tool together with a spring-dashpot model to predict particle-particle and particle-wall contact forces [26]. Specifically, the following contact force models in the tangential (i.e., $\mathbf{f}_{i, j}^{t}$ ) and normal direction (i.e., $\mathbf{f}_{i, j}^{n}$ ) were used:

$$
\begin{gathered}
\mathbf{f}_{i, j}^{n}=k^{n} \delta_{i j} \mathbf{n}_{i j}-\eta^{n} \mathbf{v}_{i j}^{n} \\
\mathbf{f}_{i, j}^{t}=-k^{t} \mathbf{u}_{i j}^{t}-\eta^{t} \mathbf{v}_{i j}^{t}
\end{gathered}
$$

with:

$$
\delta_{i j}=r_{i}+r_{j}-\left|\mathbf{r}_{i}^{\prime}-\mathbf{r}_{j}^{\prime}\right|
$$

In case of overlapping particles the normal overlap $\delta_{i j}$ is positive.

In what follows we give an overview about the most important quantities needed to interpret our work. For the meaning of the remaining symbols (which are not discussed in the text), we refer the interested reader to the Nomenclature presented at the end of our manuscript (see Section 6).

The characteristic contact time $t_{c o}$ of the above linear spring-dashpot model is defined as:

$$
t_{c o}=\pi / \omega
$$

with

$$
\omega=\sqrt{\left(k / m_{e f f}-\eta^{2}\right) /\left(4 m_{e f f}^{2}\right)}
$$

Thereby, the contact time $t_{c o}$ limits the time step $\Delta t$ for the integration of Newtons equations of motion. The coefficient of restitution $e_{n}$ is defined as

$$
e=\exp \left(\frac{-\pi \eta}{m_{e f f} \sqrt{\left(k / m_{e f f}-\eta^{2}\right) /\left(4 m_{e f f}^{2}\right)}}\right)
$$

and was adjusted to 0.9. For relevant parameter ranges and constants studied in our present work we refer to Tab. (3). We set the spring stiffness and damping coefficient in the normal and tangential direction to be identical. Specifically, we adjust $\eta$ and $k$ to obtain a certain restitution coefficient and to realize a predefined dimensionless shear rate $\gamma^{*}$. For more information on the used parameter ranges and routines to calculate, e.g., the corresponding effective Young's modulus, the reader is referred to Section 4 and Appendix A. 


\subsection{Transport within a particle}

For modeling the thermal transport inside the particle we use the simulation tool ParScale [27]. Even through ParScale is capable of resolving individual property profiles (e.g., a dissolved chemical species) within a variety of chemical and drying models (Forgber et al. [24]), we only account for individual temperature profiles of each particle in our present simulations. By using the Method of Lines (MoL), which is integrated in the CVODE integrator that forms the core of ParScale, we are enable to perform flexible and stable simulations, even in case of fast changing environmental conditions (Cohen et al. [28]). For a more detailed insight into the solution procedure, and for overview of the parallel coupling strategy we refer to Forgber et al. [25] and the comprehensive online documentation [27]. The next section summarizes the governing equations for the thermal transport inside the particle.

\subsubsection{Governing equations}

Our basic assumption is an assembly of solid and non-porous particles. The remaining thermal energy balance equation is (i) discretized in space and (ii) integrated in time using ParScale. We use a spatial discretization of each particle with a fixed radial space resolution. The differential heat balance in spherical coordinates is solved (see Eqn. (8)), assuming spherical symmetry (i.e., discretization is done using concentric spherical shells). Since we are only tracking solid, non-porous particles, we can assume for the effective heat conductivity $\lambda_{\text {eff }}=$ const. After introducing the thermal diffusivity $a$, the differential heat balances reads:

$$
\begin{gathered}
a=\frac{\lambda_{e f f}}{\rho c_{p}} \\
\frac{\partial T}{\partial t}=-\nabla \cdot\left(-a \frac{\partial T}{\partial r}\right)
\end{gathered}
$$

For discretisation in physical space a second order central differencing scheme is used. For details of the differencing scheme, as well as results of the verification studies, the reader is again referred to Forgber et al. [25] and the official documentation which is available online [27]. The next section outlines definitions of all thermal fluxes taken into account in our simulations, including their physical interpretation. These fluxes are computed with the simulated particle and contact information (e.g., particle positions, speed, as well as particle-particle and particle-wall overlaps), and hence present the main outcome of our simulations.

\subsection{Thermal Fluxes}

The definition of thermal fluxes in the present work matches that presented by Forgber et al. [25], Rognon et al. [21] and Mohan et al. [19]. When performing thermal DEM simulations Rognon et al. [21] showed that the convective flux (defined in Eqn. 9) can easily exceed the conductive flux. This is especially true for high shear rates. The convective thermal flux occurs due to the movement of the particles in the simulation domain, and takes the effective mass $m_{\text {eff }}$, the thermal capacity $c_{p}$, the volume averaged temperature $T_{v o l, a v g}$ and the velocity $\mathbf{v}_{i}$ of each particle into account. In other words - the convective thermal transport represents the redistribution of thermal energy as a result of the particle movement caused by the applied shear in the simulation domain. Note that our simulation setup is bounded by walls, and that the normal vector of these 
walls matches the direction of an applied temperature gradient. In the other directions (i.e., the shear and spanwise direction) periodic boundary conditions are applied. In earlier work (Forgber et al. [25]) the temperature gradient was enforced by different thermal regions in the simulation setup: layers of particles were set to a specific temperature which narrowed the region available for post-processing and introduced a so-called "drift-convective" flux. Since the temperature gradient is realized through a fixed wall temperature and the full simulation domain size is used for post-processing, the convective flux can be calculated as follows:

$$
\mathbf{q}^{\text {conv }}=\frac{1}{V} \sum_{i} m_{e f f, i} c_{p, i} T_{v o l, a v g, i} \mathbf{v}_{i}
$$

The conductive thermal transport (Eqn. 10) takes particle-particle interactions into account, as it relies on the summation over all collisions. Thereby the main influence parameters are the temperature differences between the particles and the overlapping area in which heat conduction occurs.

$$
\mathbf{q}^{\text {cond }}=\frac{1}{V} \sum_{C} 2 \lambda_{P} A_{c o}^{1 / 2}\left(T_{i}-T_{j}\right) \mathbf{r}_{i j}
$$

Since we assume non-vacuum conditions, we take the transmitted heat to the surrounding fluid into account. Note the the fluid has no influence on the particle movement since we assume the particles to have a high Stokes $(S t)$ number as discussed by Yin et al. [29]. It is therefore suitable to rely on a pure DEM method for the particle-particle interaction and FVM for the inner particle properties. As it is well know, the transferred thermal flux is calculated as a function of the heat transfer coefficient $\alpha$ and the temperature difference between particle temperature $T_{i}$ and environment temperature $T_{f, i}$ seen by every particle.

$$
q^{\text {trans }, i}=\alpha\left(T_{i}-T_{f, i}\right)
$$

Since our study is aimed towards a general model, we set $T_{f, i}$ to a constant value. A more detailed insight on the simulation domain and adjusted parameters is given in Sec. 4. An overview about equations solved and unknown particle quantities are given in Tab. (1) and Tab. (2), respectively. For the reference flux we choose the maximum conductive flux in case of a box fully filled with the solid material that constitutes the particles, which is hence calculated as:

$$
q_{s}=-\lambda_{e f f} \nabla y T
$$

where $\partial T / \partial y$ is the temperature gradient in the gradient direction (e.g. $y$-direction) and can be calculated as:

$$
\nabla_{y} T=\frac{T_{t o p}-T_{b o t}}{H}
$$

with $H$ representing the wall distance (i.e., the simulation domain size) and $T_{\text {top }}$ and $T_{b o t}$ denote the temperatures of the top and bottom wall, respectively. 


\begin{tabular}{ll}
\hline Reference System & Equation \\
\hline Particle flow & $\frac{d \mathbf{x}_{i}}{d t}=\mathbf{v}_{i}$ \\
& $m \frac{d \mathbf{v}_{i}}{d t}=\sum_{j}\left(\mathbf{f}_{i j}^{t}+\mathbf{f}_{i j}^{n}\right)$ \\
& $I_{i} \frac{d \boldsymbol{\omega}_{i}}{d t}=\sum_{j} \mathbf{f}_{i j}^{t} \times \mathbf{r}_{i j}$ \\
\hline Internal thermal transport & $\frac{\partial T}{\partial t}=a \cdot\left(\frac{\partial^{2} T}{\partial r^{2}}+\frac{2}{r} \frac{\partial T}{\partial r}\right)$ \\
\hline
\end{tabular}

Table 1: Covering equations for particle flow (Newtons equation of motion) and equations regarding internal thermal transport in spherical coordinates in one-dimensional form.

\begin{tabular}{ll}
\hline Reference System & Unknown quantities \\
\hline Particle flow & Angular velocity $\boldsymbol{\omega}_{i}$ \\
& Particle velocities $\mathbf{v}_{i}$ \\
& Particle positions $\mathbf{x}_{i}$ \\
\hline Internal thermal transport & Particle temperature $T$
\end{tabular}

Table 2: List of unknown simulation quantities.

\section{Theoretical Analysis}

\subsection{Dimensional Analysis}

Granular rheology is an active research field, and hence the main influencing parameters on the flow have been well documented by, e.g., Mohan et al. [19], Chialvo et al. [30] and our earlier work (Forgber et al. [25]). In agreement with the mentioned work we use the following definition for a dimensionless shear rate:

$$
\dot{\gamma}=\gamma d_{p}^{3 / 2} / \sqrt{k_{n} / \rho_{p}}
$$

A major outcome of recent simulations (Hatano et al. [5], Otsuki et al. [8], Alonso-Marroqun et al. [31]) and experiments (Nordstrom et al. [6]) are simple expressions for the contact pressure. Specifically, it is possible to collapse the scaled stress-shear rate data into curves by appropriately adjusting the critical volume fraction. Since the following scaling expressions are widely excepted in the granular rheology community, we use them as an inspiration to scale the conductive thermal flux over the dimensionless contact pressure later in our manuscript (see Section 4):

$$
\begin{aligned}
p_{Q S} & \sim\left|\phi-\phi_{c}\right|^{2 / 3} \\
p_{\text {int }} & \sim\left|\phi-\phi_{c}\right|^{0} \\
p_{\text {inert }} & \sim\left|\phi-\phi_{c}\right|^{-2} .
\end{aligned}
$$


Mohan et al. [19] found the Peclet number to be the most influential parameter for heat transport in granular materials and defined:

$$
P e=\frac{\left(d_{p} / 2\right)^{2}}{\lambda_{p} /\left(\rho_{p} c_{p}\right)} \gamma
$$

Thereby, the Peclet number relates convective and conductive heat transport within the particles in the absence of heat transfer to the ambient fluid. However, in the present work we consider an ambient fluid via thermal exchange via a fixed fluid temperature $T_{f}$ and heat transfer coefficient $\alpha$. Clearly, for that application, the Biot number is an additional dimensionless parameter, which is defined as:

$$
B i=\frac{\alpha d_{p}}{\lambda_{p}}
$$

The Biot number relates the internal to the external resistance to heat transport. Summarizing, we expect intra-particle temperature profiles for significantly large Biot numbers. The next section outlines the wall bounded sheared box set-up, and documents our results on how the Peclet and Biot number affects the heat fluxes through a granular material. 


\section{Wall Bounded Shear Flow}

In the following section we investigate the thermal behaviour of a wall bounded shear flow under well controlled conditions. By applying moving wall boundary condition to a semi periodic box driven by a homogeneous shear, a combination of LIGGGHTS and ParScale (Forgber et al. [25]) can be used to study various influence parameters. Both walls are moved into counter-clockwise directions with a constant velocity in $x$-direction. The dimensions of the box are chosen to be $H / d_{p}=15$ with a range of particle volume fractions $\left(\phi_{p}=0.50-0.64\right)$ studied. The $z$-direction serves as the span-wise direction, whereas in $x$-direction the homogeneous shear flow is driven. In $y$-direction we apply a temperature gradient by fixing the wall temperature at the top wall and keeping the corresponding bottom wall cold. Wide ranges of various parameters and their influence on the thermal fluxes are studied with an overview given in Tab. (3). To illustrate the simulation domain, Fig. 1 depicts the wall bounded box with periodic boundary conditions in the $x$ - and $z$-direction. The $y$-direction is bounded by walls with the arrows indicating the counter-clockwise applied shear.

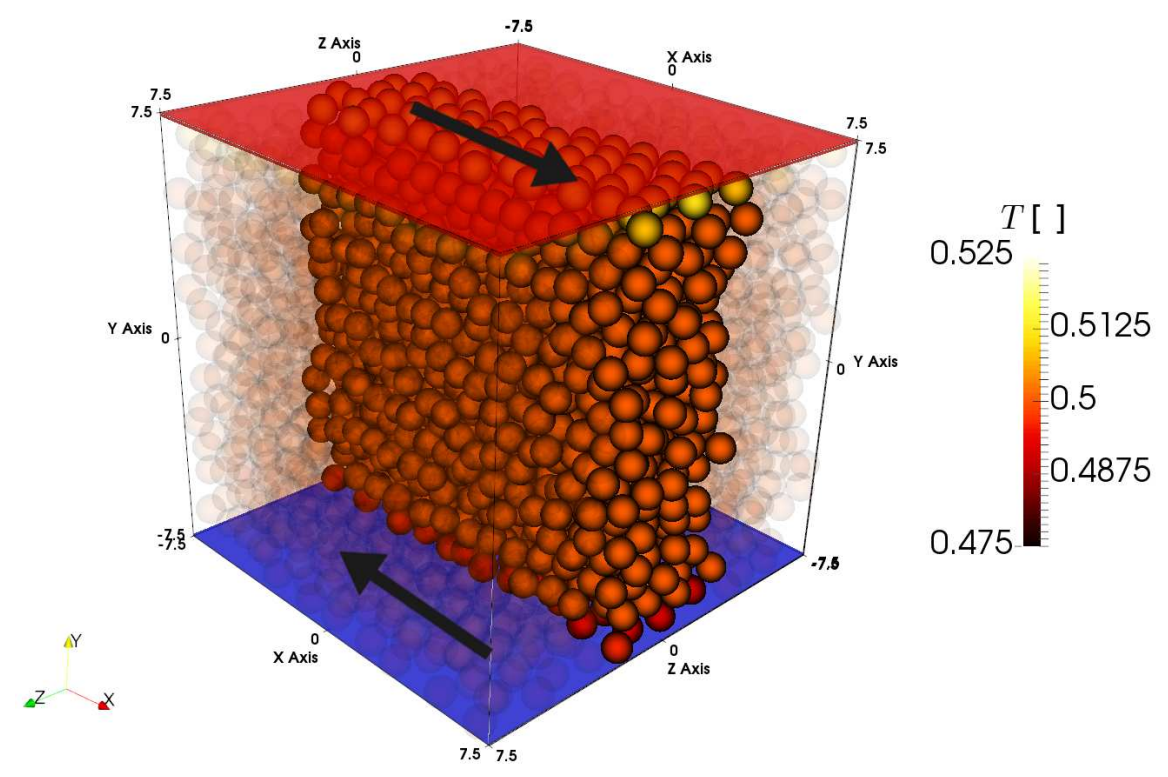

Fig. 1: Simulation set-up for wall bounded, semi-periodic sheared box simulation. Temperature gradient in $y$-direction, mean flow in $x$-direction. Black arrows indicate direction of wall movement. The transparent particles illustrate the periodic boundaries applied in the $x$ - and $z$-direction.

For all unjamming shear flow situations, the flow converges into a statistical quasi-steady state so convective and conductive fluxes through the sheared flow can be averaged and compared. Fig. 4 shows typical results from our couple LIGGGHTS and ParScale simulations. Thereby all particles are initialized with a uniform temperature, cooled down and sheared according to the studied set of dimensionless parameters. Most important, the transition between unjammed and jammed particle beds has to be distinguished from any kind of crystallization. In order to ensure this, in the next chapter we investigate crystallization systematically and provide a way to determine whether the system is crystallized or not. 
Table 3: Ranges of dimensionless parameters for wall bounded shear flow simulation.

\begin{tabular}{ccl} 
Parameter & Min. Value & Max. Value \\
\hline$\phi_{p}$ & 0.5 & 0.64 \\
$\dot{\gamma}$ & $10^{-4}$ & $10^{-1}$ \\
$P e$ & $10^{-2}$ & $10^{2}$ \\
$B i$ & 0 & 50 \\
\hline$\mu$ & 0.5 & fixed \\
$e$ & 0.9 & fixed \\
$T_{f}$ & $\left(T_{\text {top }}+T_{\text {bot }}\right) / 2$ & fixed \\
\hline
\end{tabular}

\subsection{Crystallization as a Function of the Friction Coefficient and Dimensionless Shear Rate}

The goal of this chapter is to adjust our simulation setup in order to ensure physical behaviour of the sheared granular material. In contrast to our previous study (Forgber et al. [25]) which were mostly performed within a fully-periodic box, our set-up is bounded by walls. We chose this because of (i) the amplification of jamming and crystallization by walls and (ii) the gap in literature by shifting the focus to more application-near simulations that include walls. As reported frequently in the literature (Tsai et al. [32], Liu et al. [15], Luding [16]) mono-disperse particles tend to form an ordered state, which might cause crystallisation. In the contrary, polydispersity can prevent these ordering processes. It is also well known that crystallization in poly-disperse materials with a wide range of size distribution is impossible (Herminghaus [33], [34]). Since mono-disperse, spherical particles are of subordinate role for real life applications, this phenomena is of a pure numerical nature in our simulation. It is obvious that boundary walls tend to amplify crystallization, since particles cannot pass the boundary in the span-wise direction. According to recently published regime maps, a suitable way to check for this phenomena is to screen the effect of the friction coefficient and the dimensionless shear rate. According to existing regime maps, a higher dimensionless shear rate always result in enhanced heat transfer through conduction (Rognon et al. [21], [20], Mohan et al. [19], Forgber et al. [25]). We show that this trends are not reproducible in case of crystallization by plotting the dimensionless conductive heat flux over the dimensionless shear rate for a sufficiently high particle volume fraction. We look at a dense granular flow to picture the transition where crystallization and jamming has been frequently been reported (e.g., by Tsai et al. [32]). Form Fig. (2) we observe a nearly constant conductive heat flux for all dimensionless shear rates in combination with sufficiently high friction coefficients. Taking the parameter ranges into account, we conclude that a quasi-static flow regime prevails, in which the conductive thermal transport rate, as well as the scaled contact pressure, becomes independent of the particle stiffness. This has been already demonstrated by Rognon et al. [21]. When lowering the coefficient of friction (i.e., from 0.5 to 0 ) the dimensionless conductive heat flux drops by around seven orders of magnitude. Also, for some coefficients of friction (i.e. 0.1 and 0.3 ) a transition can be observed with dimensionless heat fluxes dropping by two to six orders of magnitude. In that region we observe a partially crystallized granular flow with ordering of particles originating from both walls simultaneously. This has been documented based on simulations and experiments by Tsai et al. [32]. The time for a fully ordered system is highly depending on shear rate and box size. We did not investigate this transient behavior at this point, since we aim for the fluxes and pressures in a statistical steady state. Also, due to reproducibility of the results, we rely on a mono-sized simulation domain. Taking existing regime maps into account (see, e.g., Rognon et al. [20] , Forgber et al. [25]) a higher dimensionless 


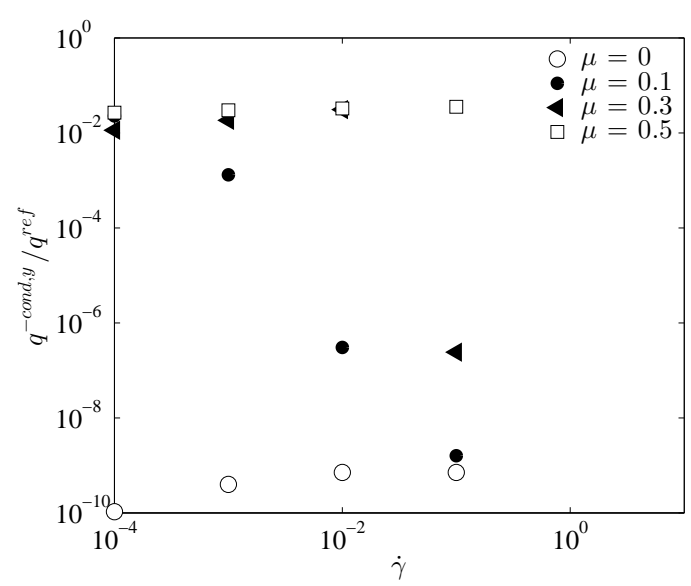

Fig. 2: Conductive heat fluxes for crystallized and non-crystallized granular materials for $P e=$ $0.01, B i=0.1$ and $\phi=0.62$.

shear rate result in greatly reduced heat transfer rates due to conduction. We conclude that this decrease is due to crystallization and therefore unwanted in numerical simulations. To support our conclusion of crystallization, Fig. 3 shows the particle velocity distribution in the main flow direction for the middle layers of spheres for the two extreme cases. Note that both snapshots were produced using the same dimensionless parameters, except for the coefficient of friction. The first panel is a disordered dense fluid where particles move and collide randomly within the

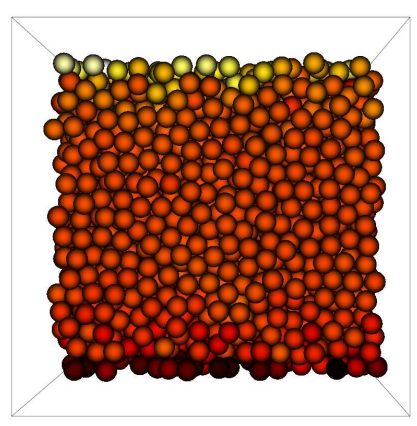

(a)

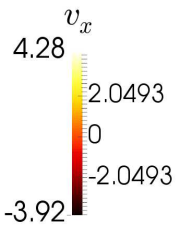

Fig. 3: Crystallized and non-crystallized granular shear flow for $P e=0.01, B i=0.1, \phi=$ $0.62, \dot{\gamma}=0.01$ and (a) $\mu=0.5$, (b) $\mu=0.0$

shear flow. The velocity magnitudes in the main flow direction indicate a fluid-like behaviour where every applied yield stress results in a deformation of the material. In the second panel each sphere is locked with its six neighbours and densely packed into a hexagonal array without possible movement of a single sphere. Fig. 3 illustrates this, where most of the spheres in the domain are stagnated. 
Clearly, the crystallization of granular material is an active research field and can give rise to complex side phenomena and transport effects ( Herminghaus [33]). Since this is not the main focus of our manuscript, we adjust our coefficient of friction to 0.5 which enables us to always ensure a non-crystallized granular shear flow in the shear cell. In the next Section, we study the influence of various flow situations on the conductive thermal transport.

\subsection{Conductive Transport in Wall Bounded Shear Flow}

First we look at the influence for a variety of dimensionless parameters on the conductive transport in wall bounded shear flows. Fig. 4 shows the dimensionless conductive heat flux over the volume fraction for $P e=0.01$ and different dimensionless shear rates (i.e., the inverse of the particle stiffness).

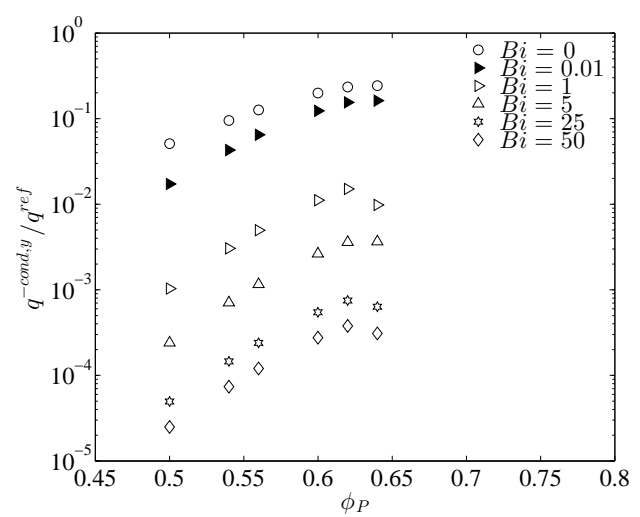

(a)

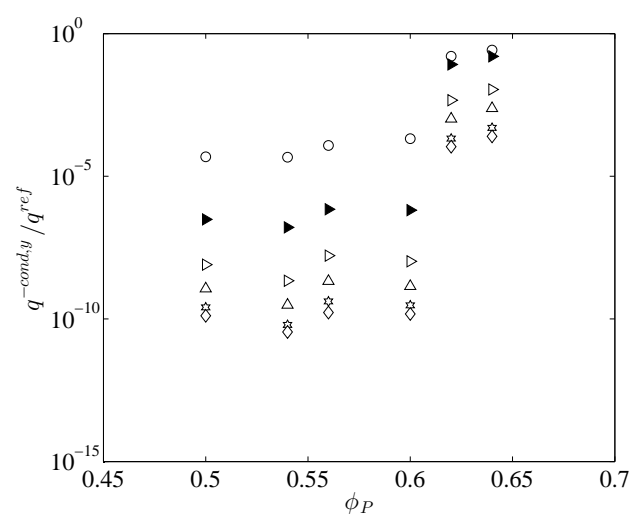

(c)

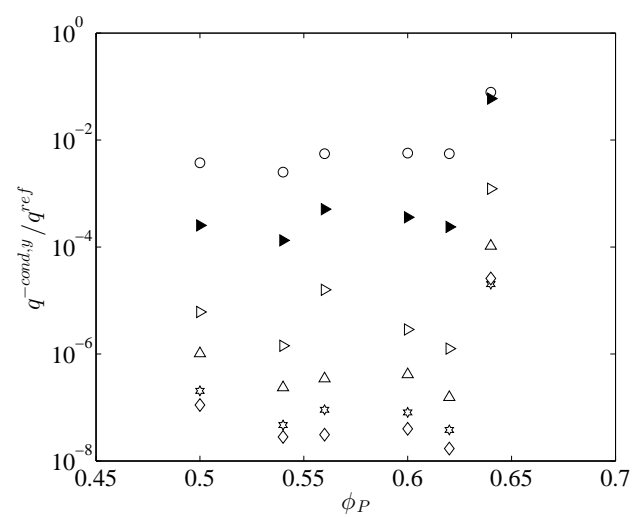

(b)

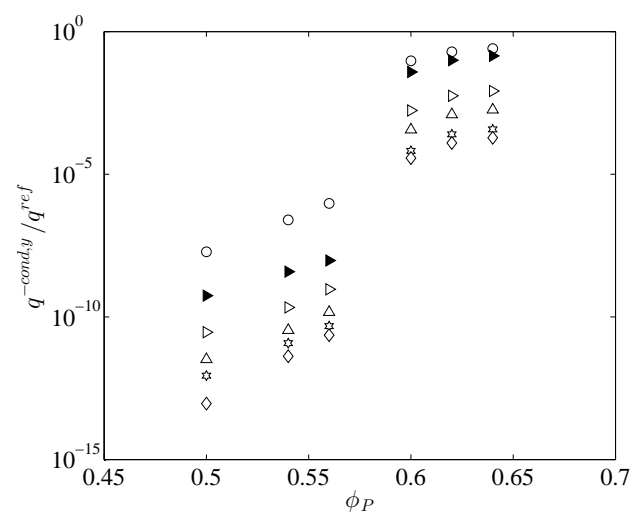

(d)

Fig. 4: Scaled conductive flux for $P e=0.01, \mu=0.5$, (a) $\dot{\gamma}=10^{-1}$, (b) $\dot{\gamma}=10^{-2}$, (c) $\dot{\gamma}=10^{-3}$, (d) $\dot{\gamma}=10^{-4}$

Fig. 4 shows a wide range of flow situations. Most importantly, it indicates a significant change in the conductive thermal transport through the sheared bed upon an increase of the particle concentration, and for which jamming occurs. It has been described frequently in literature that 
the applied set of dimensionless parameters to a sheared box simulation can change the critical particle volume fraction at which jamming appears (e.g., see Ciamarra et al. [17], as well as the original jamming diagram proposed by Liu et al. [15]). We show that this transition to a jammed state is not only accompanied by strong pressure and coordination number fluctuations (Chialvo et al. [30]), but also by an enhancement in the conductive flux by up to five orders of magnitude. Thereby the amplification of the thermal flux is mainly depending on the particle stiffness. From Fig (4) we want to point out that the particle stiffness has a significant influence on the jamming point. For very soft particles (compare Fig. 4 (a), $\dot{\gamma}=10^{-1}$ ) no jump in the conductive thermal flux can be seen and therefore our data indicates a gradual transition from an unjammed to a jammed state. As the particle stiffness is increasing (i.e., the dimensionless shear-rate is lowered) a reduction of the critical volume fraction can be seen. Fig. 4 (pane b) indicates a jammed state at $\phi_{P}=0.64$, whereas in Fig. 4 (panel c) the critical particle volume fraction drops to 0.62. For our smallest studied dimensionless shear rate (Fig. 4 (d), $\dot{\gamma}=10^{-4}$ ) we found the jamming volume fraction to be smaller than 0.6. Even though the magnitude of the scaled conductive flux varies by orders of magnitude, it can be seen our data in Appendix B and C (Fig. 9 and Fig. 10), that the critical jamming volume fraction remains constant for different shearing speeds expressed by the Peclet number. From Fig. 4 (b) and (c) we observe small fluctuation in the scaled conductive heat flux with rising volume fraction. According to theory, a higher volume fraction should lead to enhanced conductive transport since more particles result in a larger contact area. To a certain extend (i.e., in case of high $B i$ numbers) these fluctuation are also present in case of fast shearing (see our data in Appendix B and C). Since we adjusted the coefficient of friction to 0.5 , these fluctuation could be explained by the transition between shear thickening/thinning (Brown et al. [18]), or the so called "Flow and Jam" and "Slip and Jam" regimes (Ciamarra et al. [17]). Both works indicate regime changes and large pressure fluctuations around the considered volume fraction, which is directly related to the contact area between the particles, and therefore to the conductive thermal flux (compare Eqn. 10). Taking these findings and previous work on collapsing pressure, stress and shear rate curves into account, we now try to find a relation between dimensionless conductive flux and scaled contact pressure.

\subsection{Collapsed Conductive Flux over Contact Pressure}

By following the previous work by Rognon et al. [20], Chialvo et al. [30] and Olsson et al. [35] we show a way to predict the conductive flux in sheared beds in which heat is also transmitted to the environment, and in which jamming occurs. In detail, we show that the conductive flux can be collapsed ont a single curve, independent of any specific flow configuration or jamming, by plotting it against the dimensionless contact pressure. In fact Fig. 5 shows a diagram similar to Rognon et al. [20] for the scaling of eigencondutivities as a function of the solid fraction, coordination number and eigen-stresses. We found the exponent $n$ to be 0.82 which results in a reasonable fit of all data. Note that this plot collapse all data ranges mentioned in Tab. (3). The jump in the conductive flux due to jamming is not seen here since the dimensionless contact pressure is enhanced by the same order of magnitude. Our data also indicates an nearly independent behaviour of the Peclet number (compare Fig. 4, 9, 10) and the dimensionless shear rate. The scattering of the data is within the fluctuations of the conductive heat flux which allows us to find the following fitting functions:

$$
\begin{aligned}
& B i^{n} \frac{q^{- \text {cond,y }}}{q^{r e f}} \sim 0.010 p_{c} \frac{d_{p}}{k_{n}}, \quad \text { unjammed regimes } \\
& B i^{n} \frac{q^{-c o n d, y}}{q^{r e f}} \sim 0.314 p_{c} \frac{d_{p}}{k_{n}}, \quad \text { jammed regimes }
\end{aligned}
$$




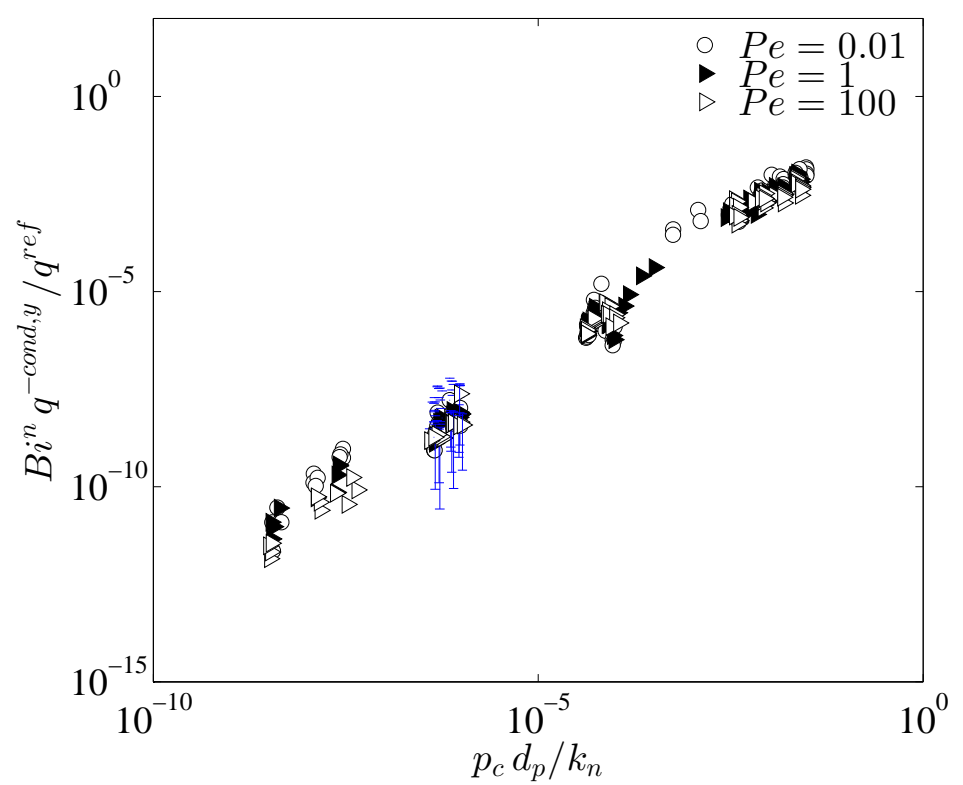

Fig. 5: Collapsed dimensionless conductive flux over dimensionless contact pressure (with $n=$ 0.82 ), as well as error bars that illustrate fluctuations of the data in the transition regime.

Due to the large variety in data (10 orders of magnitude) a single fitting function does not cover all data with sufficient accuracy. We split our data in two regimes regarding unjammed (compare Fig. 6 (panel a), including the transition regime mentioned in Sec. 4.2), and jammed (compare Fig. 6 (panel b)) states. For both regimes we find linear relationships which describe the increase of the conductive thermal flux, accounting for the transmitted heat to the ambient fluid (since we scale with $\mathrm{Bi}$ ) over the dimensionless contact pressure. Besides the fitting function, Fig. 6 includes bounds which represent the typical fluctuations of the conductive flux during shearing. We find standard deviation to be $\sim 25 \%$ averaged for all shown data. The amount is mainly caused by high variability of our data in case of a regime transition, similar to that identified by Chialvo et al. [30] for pressure fluctuations. For these situations, we also show typical fluctuations in the heat flux in Fig. 5. Furthermore, especially in fast sheared granular materials (see Fig. 6 for $P e=100)$ in combination with low packing fractions and high Biot numbers, the data differs from the fitting function (Fig. 6 (a)). For regimes where the dimensionless conductive transport is high, our correlation shows good agreement with the simulation data. Occurring differences are well inside typical fluctuations for most of the data. Nevertheless, our model should only be taken as a coarse approach to determine the transferred heat through conduction. Due to the large range of relevant dimensionless parameters studied, we anticipate that it can be widely applied to sheared beds, even in jammed states. After analysing the conductive heat transport through the granular bed, we now analyse the convective transport.

\subsection{Convective Flux over Peclet Number}

The convective heat transport mechanism is not directly related to material properties as it is mainly a function of the grain temperature and the particle velocity (see Eqn. (9)). The latter is represented by the Peclet number (i.e., a metric for the speed of shearing). Therefore, we 


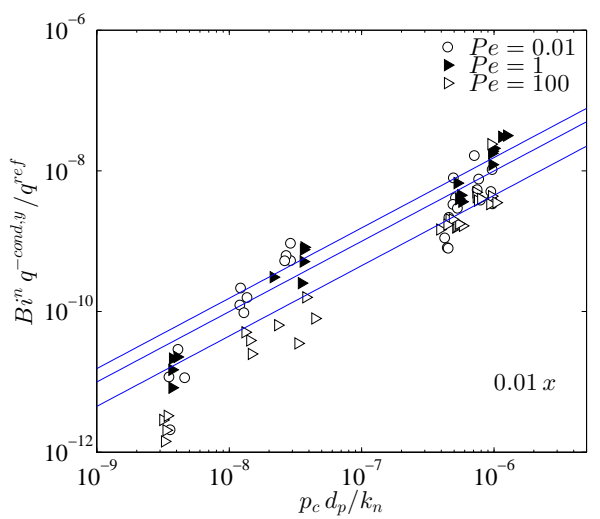

(a)

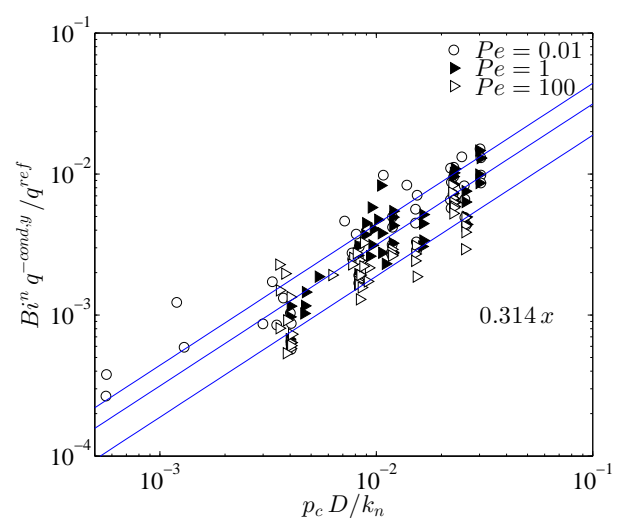

(b)

Fig. 6: Collapsed dimensionless conductive flux over dimensionless contact pressure for $n=0.82$ and (a) unjammed (b) jammed granular material. Blue lines indicate upper and lower limits for the averaged fluctuations calculated for the shown data ranges.

investigate the influence of the Peclet number on the convective flux and present the results in Fig. 4. For both Biot numbers we observe a similar behaviour as seen by Rognon et al. [21]: the

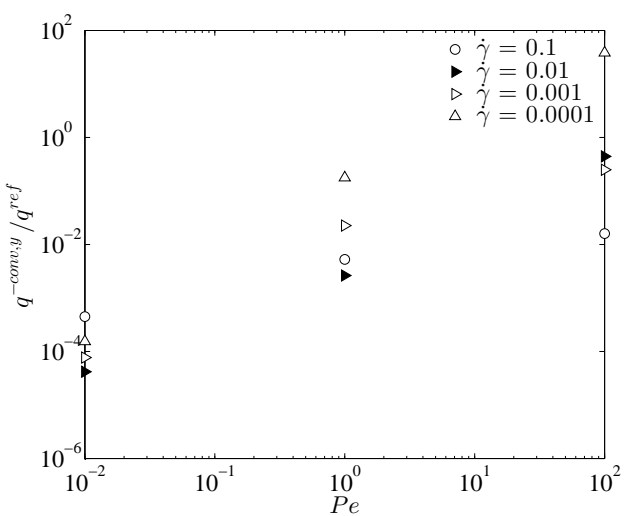

(a)

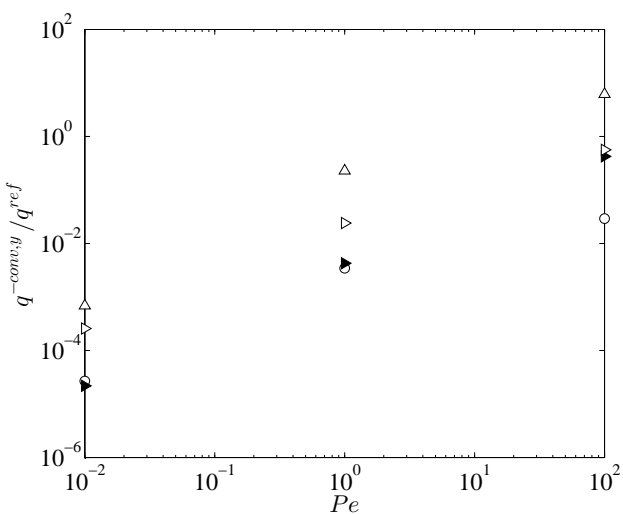

(b)

Fig. 7: Scaled convective flux for several dimensionless shear rates and Biot numbers for $\phi_{P}=$ 0.64 , (a) $B i=0$, (b) $B i=1$

convective heat flux easily exceeds the conductive heat transport rate for fast sheared beds. This is possible since the reference heat flux is defined as the heat transferred by conduction in case the simulation box would be completely filled with solid (stagnant) material. In general we agree with the findings of Rognon et al. [21] in terms of the influence of the particle stiffness. We find that stiffer particles cause an enhanced convective transport, especially for high Peclet numbers. When shifting our focus to low Pelcet numbers, the convective transport of soft particles might exceed that of the stiffer ones, which is also in good agreement with Rognon et al. [21]. This previous study found the correlation $q_{c o n v, y}=10^{-2} q_{s} \tau$, which holds for sufficiently large thermal numbers $\tau$. Furthermore, no drastic change of the convective flux is observed, as illustrated in 
Fig. 7. This is in contrast to the observed jump in the conductive thermal transport rate when transitioning from the unjammed to the jammed state. Our findings from Section 4.2 indicate that the jump is independent of the Peclet number, and the main dependency is with respect to the critical volume fraction and dimensionless shear rate. To prove our theory that the convective flux is not affected by jamming of granular material a trend for different volume fractions is needed.

\subsection{Convective Flux over Particle Concentration}

In order to provide a meaningful comparison to the conductive flux enhancement in case of particle jamming (compare Fig. 4), we considered the convective thermal transport for different packing fractions. Note that for the conductive heat flux a jump by up to five orders of magnitude was observed, nearly independent of the Peclet and Biot number. Fig. 8 illustrates a weak

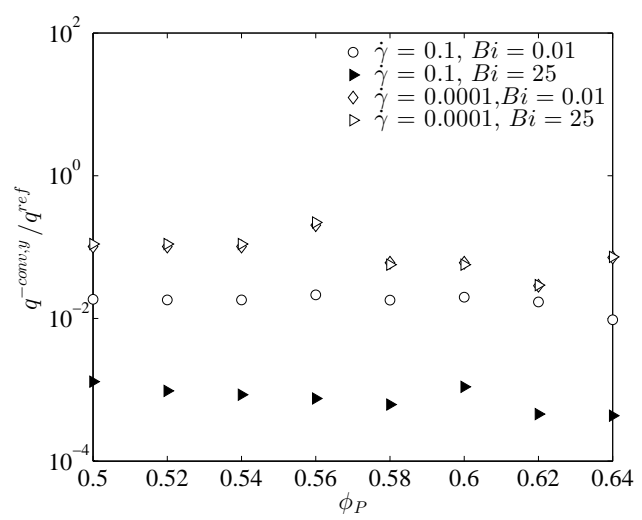

(a)

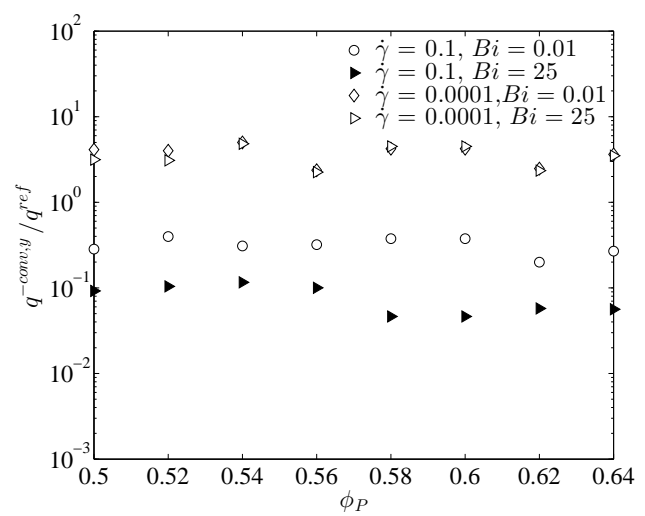

(b)

Fig. 8: Scaled convective flux versus volume fraction for four extreme cases regarding dimensionless shear rate and Biot number (a) $P e=1$, (b) $P e=100$.

dependency of the convective flux on the particle concentration, and jamming does not lead ot a substantial change in this trend. This is true for all Peclet numbers and dimensionless shear rates studied. Even though Fig. 8 only shows extreme cases regarding Biot number and dimensionless shear rate, we observe that the convective thermal transport remains nearly constant over the volume fraction. For stiff particles (e.g. $\dot{\gamma}=10^{-4}$ ) the convective transport becomes independent of the Biot number as data points for $B i=0.01$ and $B i=25$ collapse. The independence is also true for $P e=100$ (Fig. 8 (b)). In other words - the transferred heat has a negligible influence on the amount of redistributed thermal heat due to the movement of the particles. When shifting our focus to softer materials $(\dot{\gamma}=0.1)$ the independence is not present anymore since for both Peclet numbers a consequently higher convective transport is achieved for lower Biot numbers. The difference is quite small in case of fast shearing $(P e=100)$, but increases up to two orders of magnitude for slow shearing $(P e=1)$. We conclude that for stiff particles the convective thermal transport becomes mainly a function of the particle velocity since a minor dependency on the Biot number is observed. It is clear, that higher particle velocities are seen for stiffer particle suspensions since a higher contact pressure is achieved in case of collisions. 


\section{Conclusions}

In the present manuscript we investigated different thermal transport mechanisms in a wall bounded sheared bed filled with mono-sized spheres. Thereby, we couple the library ParScale to an existing DEM solver (LIGGGHTS) in order to resolve particle-internal temperature profiles. In our Discrete Element Method approach, we take (i) conductive (ii) convective and (iii) transferred heat to an ambient fluid into account. Again, we want to point out, that we have not solved any equation for the fluid phase since the coupling is realized through a fixed fluid temperature and a predefined heat transfer coefficient to realize defined cooling conditions. We then ensure that our system is not affected by any kind of ordering mechanism (i.e., crystallization), which is enhanced by the presence of walls. We present a new way of determining whether an ordering occurs or not by considering the behavior of the conductive heat transfer rate as a function of the shear rate. We come to the conclusion that systems of mono-sized spheres reach the ordering state later in case of higher friction coefficients, consequently adjust the coefficient of friction to 0.5 in our setup to prevent crystallization. In the following we show a conductive flux enhancement up to five orders of magnitude in case of jamming, which is due to the strong increase of the contact pressure upon an increase of the particle concentration.

Furthermore, our results indicate an independence of the critical volume fraction for the increase of the conductive flux on the Peclet number (i.e., the speed of shearing). Again, this suggest that this increase of the heat flux originates from the increase of the contact pressure. Due to this finding, and inspired by the work of Rognon et al. [21] and Chialvo et al. [30], we identify scaling laws for the conductive heat flux and a possible collapse over the dimensionless contact pressure. We distinguish between jammed and unjammed regions, and present simple correlations which, most important, take the transferred heat to the ambient fluid into account. When shifting our focus to the convective thermal transport, no direct relationship between thermal flux and critical volume fraction can be observed. The convective thermal transport rate remains mostly constant for different packing fractions. By varying the Biot number we point out that the transferred heat to the fluid has no influence on the convective flux in case of stiff particles. We conclude that in this case the convective flux is mainly a function of the speed of shearing as the data collapse into one point. In case of softer materials a clear dependence is seen, and the transferred heat to the ambient fluid cannot be neglected anymore. This is indicated by a clear dependence of the convective heat flux, i.e., a variation up to two orders of magnitude, on the Biot number. Evaluated over the Peclet number, the convective thermal transport can easily exceed the conductive thermal transport rate, independent of the particle volume fraction and the Biot number. We find, in agreement with Rognon et al. [21], that the convective flux benefits from the presence of stiff particles since it is significantly higher. Also, our data agrees with the scaling law proposed by Rognon et al. [21], which showed that $q_{c o n v, y} \sim 10^{-2} q_{s} \tau$ where $\tau$ represents a thermal number similar to our Peclet number.

Future studies should focus on more detailed investigations of the effect of the transferred heat flux on the convective flux. The latter appears to be the leading heat transfer mechanism in fast sheared granular beds, and hence should be modeled in greater detail. Also, the present study was not concerned with heat transfer from the walls to the ambient fluid. 
Acknowledgements We acknowledge the support by the European Commission through FP7 Grant agreement no. 604656, project 'NanoSim'. We thank 'NAWI Graz' by providing access to dcluster.tugraz.at.

Conflict of Interest: The authors declare that they have no conflict of interest. 


\section{Nomenclature}

6.1 Latin Characters

\begin{tabular}{lll}
\hline Variable & Unit & Meaning \\
\hline$a$ & $\mathrm{~m}^{2} \mathrm{~s}^{-1}$ & Thermal diffusivity \\
$A$ & $\mathrm{~m}^{2}$ & Cross sectional area \\
$B i$ & & Biot Number \\
$c_{p}$ & $\mathrm{~J} \mathrm{~kg}^{-3} \mathrm{~K}^{-1}$ & Heat capacity \\
$C$ & & Number of contacts \\
$d$ & $\mathrm{~m}$ & Diameter \\
$e$ & & Coefficient of restitution \\
$\mathbf{f}$ & $\mathrm{N}$ & Force on particle \\
$I$ & $\mathrm{~N} \mathrm{~s}$ & Moment of inertia \\
$I$ & & Inertia number \\
$k$ & $\mathrm{~N} \mathrm{~m}{ }^{-1}$ & Spring stiffness \\
$m$ & $\mathrm{~kg}$ & Mass \\
$n$ & & Number of particles \\
$P e$ & & Peclet number \\
$q$ & $\mathrm{~W} \mathrm{~m}^{-2}$ & Heat flux \\
$r$ & $\mathrm{~m}$ & Particle radius \\
$t$ & $\mathrm{~s}$ & Time \\
$T$ & $\mathrm{~K}$ & Temperature \\
$\mathbf{v}$ & $\mathrm{m} \mathrm{s}$ & Particle velocity \\
$V$ & $\mathrm{~m}^{3}$ & Volume \\
$Y$ & $\mathrm{~N} \mathrm{~m}^{-2}$ & Young's modulus \\
$\mathbf{x}$ & $\mathrm{m}$ & Particle position \\
\hline
\end{tabular}


6.2 Greek Characters

\begin{tabular}{|c|c|c|}
\hline Variable & Unit & Meaning \\
\hline$\alpha$ & $\mathrm{W} \mathrm{m} \mathrm{m}^{-2} \mathrm{~K}^{-1}$ & Heat transfer coefficient \\
\hline$\delta$ & $\mathrm{m}$ & Overlap distance during a particleparticle contact \\
\hline$\dot{\gamma}$ & & Dimensionless shear rate \\
\hline$\gamma$ & $s^{-1}$ & Shear rate \\
\hline$\mu$ & & Friction coefficient \\
\hline$\eta$ & $\mathrm{Ns} \mathrm{m}^{-1}$ & Viscous damping coefficient \\
\hline$\omega$ & $\mathrm{s}^{-1}$ & Eigen frequency of damped harmonic oscillator \\
\hline$\theta$ & $\mathrm{K}$ & Temperature difference, Scalar Quantity \\
\hline$\phi$ & & Particle volume fraction \\
\hline$\lambda$ & $\mathrm{W} \mathrm{m}^{-1} \mathrm{~K}^{-1}$ & Thermal conductivity \\
\hline$\rho$ & $\mathrm{kg} \mathrm{m}^{-3}$ & Density \\
\hline
\end{tabular}

6.3 Superscripts

\begin{tabular}{ll}
\hline Variable & Meaning \\
\hline conv & Convection \\
cond & Conductive \\
$n$ & Normal direction \\
$t$ & Tangential direction \\
trans & Transferred \\
$x, y, z$ & Direction \\
\hline
\end{tabular}

6.4 Subscripts 


\begin{tabular}{ll}
\hline Variable & Meaning \\
\hline 0 & Initial state \\
avg & Average Value \\
bot & Bottom \\
$c$ & Critical Value \\
$c h a r$ & Characteristic value \\
$c o$ & Contact \\
$e f f$ & Effective value \\
$f$ & Fluid \\
$i, j$ & Particle index \\
$i n t$ & Intermediate \\
$p$ & Particle \\
$Q S$ & Quasi-static \\
$t o p$ & Top \\
$s$ & Summation \\
$v o l$ & Volumetric Value \\
$x, y, z$ & Directions \\
\hline
\end{tabular}

\section{Appendix}

Appendix A: DEM parameters and calculation routines

The normal spring stiffness and the coefficient of restitution were set by adjusting the effective Young's modulus $Y_{\text {eff }}$ and the damping coefficient $\eta$. Thus, the following equations were applied:

$$
\begin{gathered}
k=\frac{16}{15} \sqrt{r_{\text {eff }}} Y_{\text {eff }}\left(\frac{15 m_{\text {eff }} v_{\text {char }}^{2}}{16 \sqrt{r_{\text {eff }}} Y_{\text {eff }}}\right)^{1 / 5}, \\
Y_{\text {eff }}=\left[\frac{\left(\gamma / \gamma^{*}\right)^{2} d_{p}^{3} \rho_{p}}{(16 / 15)^{4 / 5}\left(r_{\text {eff }}\right)^{2 / 5}\left(m_{e f f}\right)^{1 / 5}\left(v_{\text {char }}\right)^{2 / 5}}\right]^{5 / 4}, \text { and } \\
\eta=\sqrt{\frac{4 m_{e f f} k}{1+\left(\frac{\pi}{\ln (e)}\right)}}
\end{gathered}
$$

Note that the characteristic impact velocity $v_{\text {char }}$ drops out of Eqn. (22) after inserting Eqn. (23) and therefore is irrelevant for the subsequent dimensional analysis. Tab. 4 outlines the adjusted parameter in order to obtain the dimensionless shear rate $\dot{\gamma}$. 
Table 4: Adjusted DEM parameters.

\begin{tabular}{lll}
\hline$\dot{\gamma}$ & $Y_{\text {eff }}$ & $\triangle t$ \\
$10^{-1}$ & $1.3210^{3}$ & $2.5010^{-3}$ \\
$10^{-2}$ & $4.1810^{5}$ & $2.5010^{-4}$ \\
$10^{-3}$ & $1.3210^{8}$ & $2.5010^{-5}$ \\
$10^{-4}$ & $4.1810^{10}$ & $2.5010^{-6}$ \\
\hline
\end{tabular}

Appendix B: Conductive Thermal Flux for other Peclet numbers

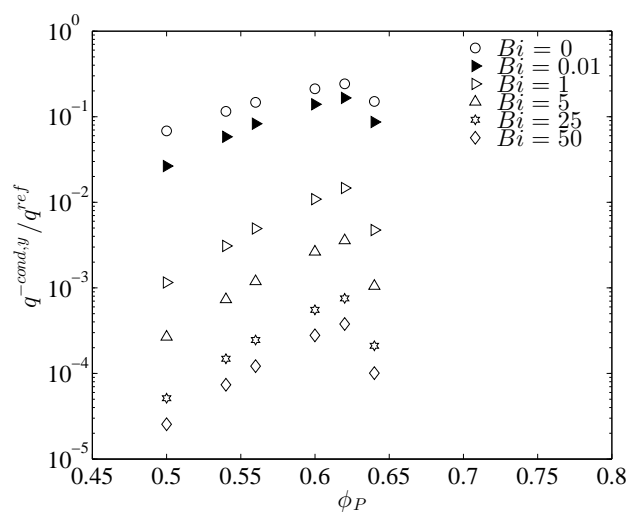

(a)

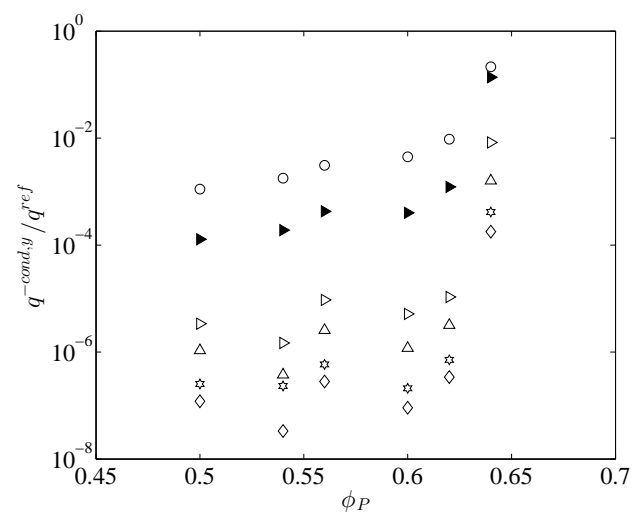

(b)

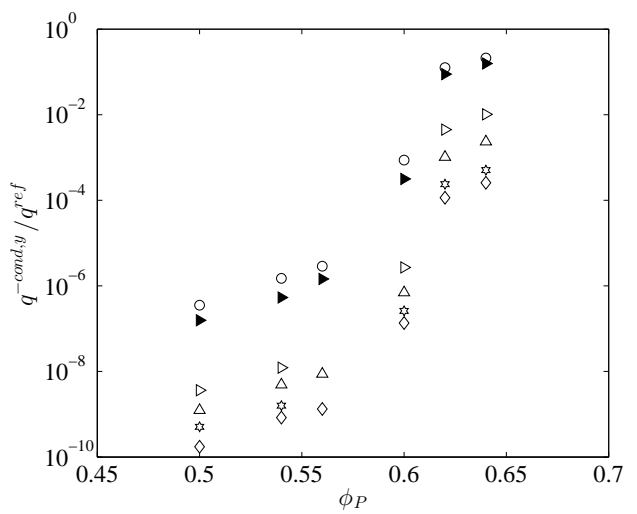

(c)

Fig. 9: Scaled conductive flux for $\mathrm{Pe}=1, \mu=0.5$, (a) $\dot{\gamma}=10^{-1}$, (b) $\dot{\gamma}=10^{-2}$, (c) $\dot{\gamma}=10^{-3}$.

Fig. 9 and 10 show a wide range of flow situations and indicate a significant change in the conductive thermal transport through the sheared bed.

Appendix C: Convective Thermal Flux for other Biot numbers 


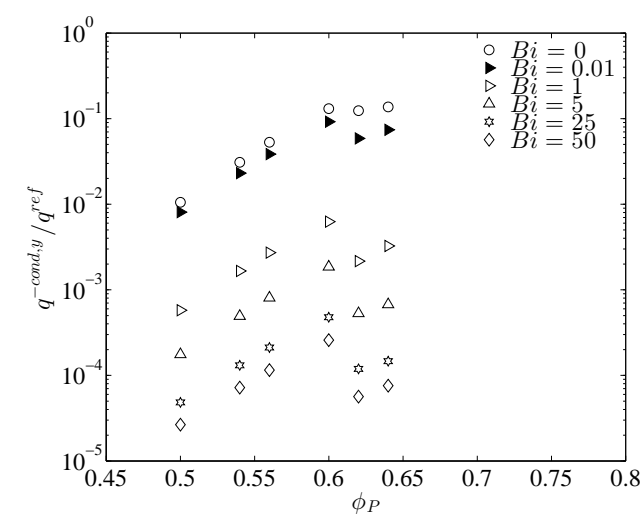

(a)

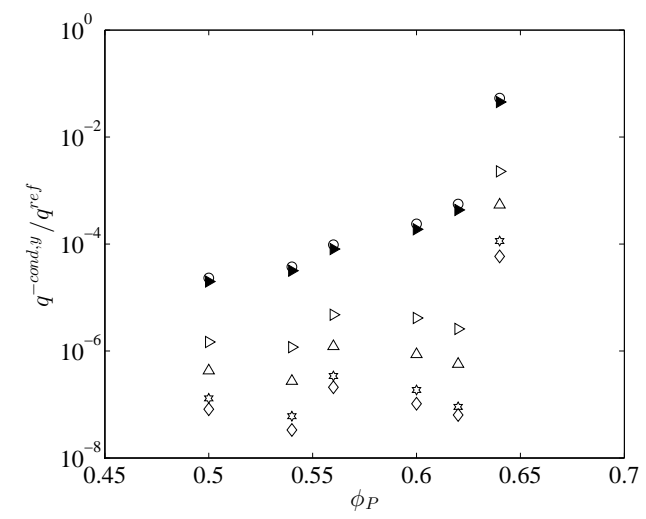

(b)

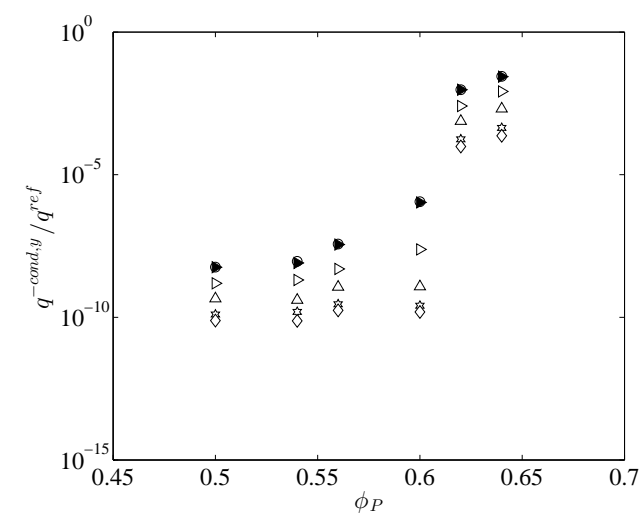

(c)

Fig. 10: Scaled conductive flux for $\mathrm{Pe}=100, \mu=0.5$, (a) $\dot{\gamma}=10^{-1}$, (b) $\dot{\gamma}=10^{-2}$, (c) $\dot{\gamma}=10^{-3}$.

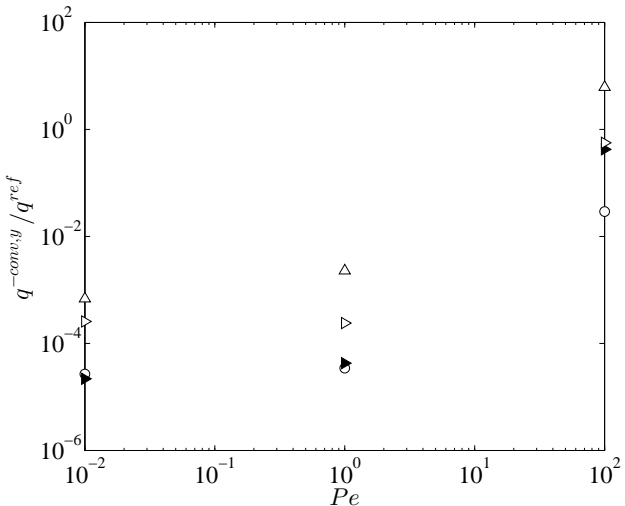

(a)

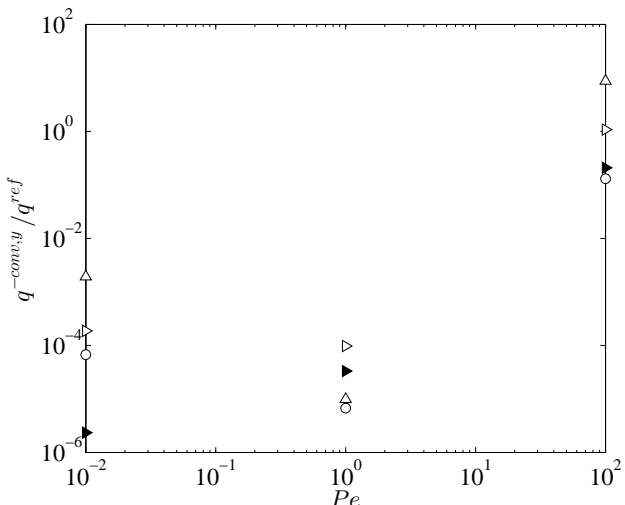

(b)

Fig. 11: Scaled convective flux for several dimensionless shear rates and Biot numbers for $\phi_{P}=$ 0.64, (a) $B i=10$, (b) $B i=50$ 


\section{References}

1. V. Garzó and J. W. Dufty. Dense fluid transport for inelastic hard spheres. Phys. Rev. E, 59:5895-5911, May 1999.

2. C. Lun, S. Savage, D. J. Jeffrey, and N. Chepurniy. Kinetic theories for granular flow: inelastic particles in couette flow and slightly inelastic particles in a general flowfield. J. Fluid Mech, 140:223-256, 1984.

3. J. T. Jenkins and M. W. Richman. Kinetic theory for plane flows of a dense gas of identical, rough, inelastic, circular disks. Physics of Fluids, 28(12):3485-3494, 1985.

4. P. C. Johnson and R. Jackson. Frictional-collisional constitutive relations for granular materials, with application to plane shearing. Journal of Fluid Mechanics, 176:67-93, 31987.

5. T. Hatano. Scaling properties of granular rheology near the jamming transition. Journal of the Physical Society of Japan, 77(12):18-21, 2008.

6. K. N. Nordstrom, E. Verneuil, P. E. Arratia, a. Basu, Z. Zhang, a. G. Yodh, J. P. Gollub, and D. J. Durian. Microfluidic rheology of soft colloids above and below jamming. Physical Review Letters, 105(17):1-4, 2010.

7. J. R. Seth, M. Cloitre, and R. T. Bonnecaze. Influence of short-range forces on wall-slip in microgel pastes. Journal of Rheology (1978-present), 52(5):1241-1268, 2008.

8. M. Otsuki and H. Hayakawa. Critical behaviors of sheared frictionless granular materials near the jamming transition. Physical Review E - Statistical, Nonlinear, and Soft Matter Physics, 80(1):5-10, 2009.

9. K. Otsuki. Fluidization and melting of fault gouge during seismic slip: Identification in the Nojima fault zone and implications for focal earthquake mechanisms. Journal of Geophysical Research, 108(B4), 2003.

10. H. W. Zhang, Q. Zhou, H. L. Xing, and H. Muhlhaus. A DEM study on the effective thermal conductivity of granular assemblies. Powder Technology, 205(1-3):172-183, 2011.

11. C. Song, P. Wang, and H. Makse. A phase diagram for jammed matter. Nature, 453(7195):629-632, 2008.

12. T. S. Majmudar, M. Sperl, S. Luding, and R. P. Behringer. Jamming transition in granular systems. Phys. Rev. Lett., 98:058001, Jan 2007.

13. B. P. Tighe, E. Woldhuis, J. J. C. Remmers, W. van Saarloos, and M. van Hecke. Model for the scaling of stresses and fluctuations in flows near jamming. Phys. Rev. Lett., 105:088303, Aug 2010.

14. D. Vescovi and S. Luding. Merging fluid and solid granular behavior. Soft Matter, 12:8616-8628, 2016.

15. A. J. Liu and S. R. Nagel. Nonlinear dynamics: Jamming is not just cool any more. Nature, 396(6706):21-22, Nov 1998.

16. S. Luding. Granular matter: So much for the jamming point. Nat Phys, 12(6):531-532, jun 2016.

17. M. P. Ciamarra, R. Pastore, M. Nicodemi, and A. Coniglio. Jamming phase diagram for frictional particles. Phys. Rev. E, 84:041308, Oct 2011.

18. E. Brown and H. M. Jaeger. Dynamic jamming point for shear thickening suspensions. Phys. Rev. Lett., 103:086001, Aug 2009.

19. B Mohan, C Kloss, J Khinast, and S Radl. Regimes of Liquid Transport through Sheared Beds of Inertial Smooth Particles. Powder Technology, 264:377-395, 2014

20. P. Rognon, I. Einav, J. Bonivin, and T. Miller. A scaling law for heat conductivity in sheared granular materials. EPL (Europhysics Letters), 89(5):1-6, Mar 2010.

21. P. Rognon and I. Einav. Thermal transients and convective particle motion in dense granular materials. Phys. Rev. Lett., 105:218301, Nov 2010.

22. A. W. Lees and S. F. Edwards. The computer study of transport processes under extreme conditions. J. $\backslash$ Phys. C - Solid, 5:1920-1921, 1972.

23. S. Radl, T. Forgber, A. Aigner, and C. Kloss. ParScale - An Open-Source Library for the Simulation of Intra-Particle Heat and Mass Transport Processes in Coupled Simulations. IV International Conference on Particle-based Methods Fundamentals and Applications (PARTICLES 2015), pages 46-55, 2015.

24. T. Forgber, J. R. Tolchard, A. Zaabout, P. I. Dahl, and S. Radl. Optimal Particle Parameters for CLC and CLR Processes - Predictions by Intra-Particle Transport Models and Experimental Validation. IV International Conference on Particle-based Methods Fundamentals and Applications (PARTICLES 2015), pages 101-111, 2015.

25. T. Forgber, B. Mohan, C. Kloss, and R. Radl. Heat transfer rates in sheared beds of inertial particles at high biot numbers. submitted to: Granular Matter, 2016.

26. C Kloss, C Goniva, A Hager, S Amberger, and S Pirker. Models, algorithms and validation for opensource DEM and CFD-DEM. Progress in Computational Fluid Dynamics, 12:140-152, 2012.

27. https://github.com/CFDEMproject/ParScale-PUBLIC, accessed September 2016.

28. S. D. Cohen, A. C. Hindmarsh, and P. F. Dubois. CVODE, A Stiff/Nonstiff ODE Solver in C. Computers in Physics, 10:138-148, 1996.

29. X. Yin and S. Sundaresan. Drag law for bidisperse gassolid suspensions containing equally sized spheres. $n d$. Eng. Chem. Res., 48:227-241, 2009.

30. S. Chialvo, J. Sun, and S. Sundaresan. Bridging the rheology of granular flows in three regimes. Physical Review E - Statistical, Nonlinear, and Soft Matter Physics, 85(2):1-8, 2012. 
31. F. Alonso-Marroquín, I. Vardoulakis, H. J. Herrmann, D. Weatherley, and P. Mora. Effect of rolling on dissipation in fault gouges. Physical Review E - Statistical, Nonlinear, and Soft Matter Physics, 74(3):1-10, 2006

32. J.-C. Tsai, G. A. Voth, and J. P. Gollub. Internal granular dynamics, shear-induced crystallization, and compaction steps. Phys. Rev. Lett., 91:064301, Aug 2003.

33. S. Strauch and S. Herminghaus. Wet granular matter: a truly complex fluid. Soft Matter, 8(32):8271-8280, 2012.

34. S. Herminghaus. Wet Granular Matter: A Truly Complex Fluid. Series in soft condensed matter. World Scientific, 2013.

35. P. Olsson and S. Teitel. Critical scaling of shear viscosity at the jamming transition. Physical Review Letters, $99(17): 1-4,2007$. 